\title{
Improving image quality in compressed ultrafast photography with a space- and intensity- constrained reconstruction algorithm
}

Liren Zhu, Yujia Chen, Jinyang Liang, Liang Gao, Cheng Ma, et al.

Liren Zhu, Yujia Chen, Jinyang Liang, Liang Gao, Cheng Ma, Lihong V. Wang, "Improving image quality in compressed ultrafast photography with a space- and intensity-constrained reconstruction algorithm," Proc. SPIE 9720, High-Speed Biomedical Imaging and Spectroscopy: Toward Big Data Instrumentation and Management, 972008 (7 March 2016); doi: $10.1117 / 12.2215017$

SPIE. Event: SPIE BiOS, 2016, San Francisco, California, United States 


\title{
Improving image quality in compressed ultrafast photography with a space- and intensity-constrained reconstruction algorithm
}

\author{
Liren Zhu ${ }^{\mathrm{a}}$, Yujia Chen ${ }^{\mathrm{a}}$, Jinyang Liang ${ }^{\mathrm{a}}$, Liang Gao ${ }^{\mathrm{b}}$, Cheng $\mathrm{Ma}^{\mathrm{a}}$, and Lihong V. Wang ${ }^{\mathrm{a}}$, \\ ${ }^{a}$ Optical Imaging Laboratory, Department of Biomedical Engineering, Washington University in St. \\ Louis, 1 Brookings Drive, St. Louis, MO USA 63130; ${ }^{b}$ Department of Electrical and Computer \\ Engineering, University of Illinois at Urbana-Champaign, 405 North Mathews Avenue, Urbana, IL \\ USA 61801
}

\begin{abstract}
The single-shot compressed ultrafast photography (CUP) camera is the fastest receive-only camera in the world. In this work, we introduce an external CCD camera and a space- and intensity-constrained (SIC) reconstruction algorithm to improve the image quality of CUP. The CCD camera takes a time-unsheared image of the dynamic scene. Unlike the previously used unconstrained algorithm, the proposed algorithm incorporates both spatial and intensity constraints, based on the additional prior information provided by the external CCD camera. First, a spatial mask is extracted from the time-unsheared image to define the zone of action. Second, an intensity threshold constraint is determined based on the similarity between the temporally projected image of the reconstructed datacube and the time-unsheared image taken by the external CCD. Both simulation and experimental studies showed that the SIC reconstruction improves the spatial resolution, contrast, and general quality of the reconstructed image.
\end{abstract}

Keywords: Ultrafast imaging, computational imaging, compressed sensing, image reconstruction

\section{INTRODUCTION}

The development of high-speed imaging technologies can be traced back over a century, to Talbot's recording of a spinning disc in the 1850s. With the advances in CCD and CMOS sensor technologies, the acquisition speed has been increased from $\sim 50$ frames per second (fps), achieved by an intermittent camera ${ }^{1}$, to one billion frames per second with a limited number of frames ${ }^{2}$. However, achieving even faster acquisition speed with these electronic sensors is impeded by the on-chip storage and electronic readout speed. The advent of the streak camera breaks this speed limit at the expense of the imaging dimensions - the use of a narrow entrance slit restrains its imaging to one spatial dimension.

Multiple techniques have been reported to enable two-dimensional (2D) ultrafast imaging. Currently, the predominant approach for capturing transient events is the pump-probe technique ${ }^{3-5}$, which has been widely used in physical chemistry $^{6}$, materials science ${ }^{7}$, and nonlinear optics ${ }^{8}$. However, the requirement of the transient events being precisely repeatable hinges pump-probe approaches' utility. Single-shot ultrafast 2D imaging techniques have been developed ${ }^{9-11}$. The sequentially timed all-optical mapping photography (STAMP) uses burst illumination produced by a spatiotemporally modulated ultra-short laser pulse to enable trillion-frames-per-second photography with a sequential depth of up to six frames. However, its reliance on the specialized active illumination rules out imaging of luminescent objects. Recently efforts have been made to utilize the streak camera as a 2D imager. Notable approaches include using a tilted lenslet array ${ }^{12}$ or a $2 \mathrm{D}$ pinhole-array ${ }^{13}$ to achieve parallel streak framing. However, these methods suffer from significant hardware light throughput loss in the pursuit of higher frame rates.

To overcome these limitations, we developed compressed ultrafast photography (CUP), a new computational ultrafast imaging technology that can capture transient dynamic events at 100 billion fps in a single measurement with a sequence depth of hundreds of frames ${ }^{14}$. CUP synergistically combines two technologies: the streak camera and compressed sensing (CS). Unlike other streak-camera-based ultrafast imagers, in a CUP camera, the entrance slit of the streak camera is fully open. In addition, the dynamic scene is spatially encoded with a pseudorandom-binary code through a digital micromirror

*Corresponding author: LHWANG@WUSTL.EDU

High-Speed Biomedical Imaging and Spectroscopy: Toward Big Data Instrumentation and Management, edited by Kevin K. Tsia, Keisuke Goda, Proc. of SPIE Vol. 9720, 972008 • ( 2016 SPIE CCC code: $1605-7422 / 16 / \$ 18 \cdot$ doi: $10.1117 / 12.2215017$ 
device (DMD). Provided the spatiotemporal sparsity of the dynamic scene, which holds in many if not most natural scenes, a CS-based reconstruction algorithm can successfully decode the spatiotemporal mixing in the vertical axis of the streak camera and retrieve spatiotemporal information.

Using CUP, we have visualized many transient light-speed phenomena ${ }^{14}$, including the propagation, reflection, and refraction of a short laser pulse in space, faster-than-light propagation of non-information, and color-resolved fluorescent excitation and emission. Recently, by leveraging the time-of-flight (ToF) of light signals backscattered from a threedimensional object, CUP has also been used for dynamic volumetric imaging ${ }^{15}$.

Currently, CUP relies on the unconstrained two-step iterative shrinkage/thresholding (TwIST) algorithm ${ }^{16}$ to reconstruct the event datacube. The reconstructed image resolution is degraded by approximately a factor of two, due to the temporal shearing operation in the streak camera and reconstruction. In this work, we report the incorporation of additional prior information from an external CCD camera, which records a time-unsheared image of the dynamic scene. With the superior spatial resolution provided by the external CCD, we formulate a space- and intensity-constrained (SIC) reconstruction algorithm to fully utilize this additional view of the scene, with the goals of improving image resolution, mitigating low-intensity artifacts, and boosting the general image quality of CUP.

\section{RECONSTRUCTION ALGORITHM}

In the conventional CUP system, the observed dynamic scene is spatially encoded by the DMD, temporally sheared by the streak tube, and then spatiotemporally integrated by the internal CCD camera inside the streak camera. As discussed previously $^{14}$, to reconstruct the original scene, one needs to solve the following inverse problem:

$$
\widehat{\mathbf{x}}=\arg \min _{\mathbf{x}}\left\{\frac{1}{2}\|\mathcal{T} \mathcal{S C}(\mathbf{x})-\mathbf{y}\|_{2}^{2}+\lambda\|\mathbf{x}\|_{\mathrm{TV}}\right\},
$$

where $\mathbf{x}$ is the intensity distribution of the dynamic scene, $\mathcal{T}$ is the spatiotemporal integration operator, $\mathcal{S}$ is the temporal shearing operator, $\mathcal{C}$ is the encoding operator that comes from the DMD, $\mathbf{y}$ is the streak camera measurement, $\|\cdot\|_{2}$ denotes the $\ell^{2}$ norm, $\|\cdot\|_{\mathrm{TV}}$ denotes the total variation (TV) norm, and $\lambda$ is the regularization parameter that tunes the ratio between the measurement fidelity term and the TV-based regularization term.

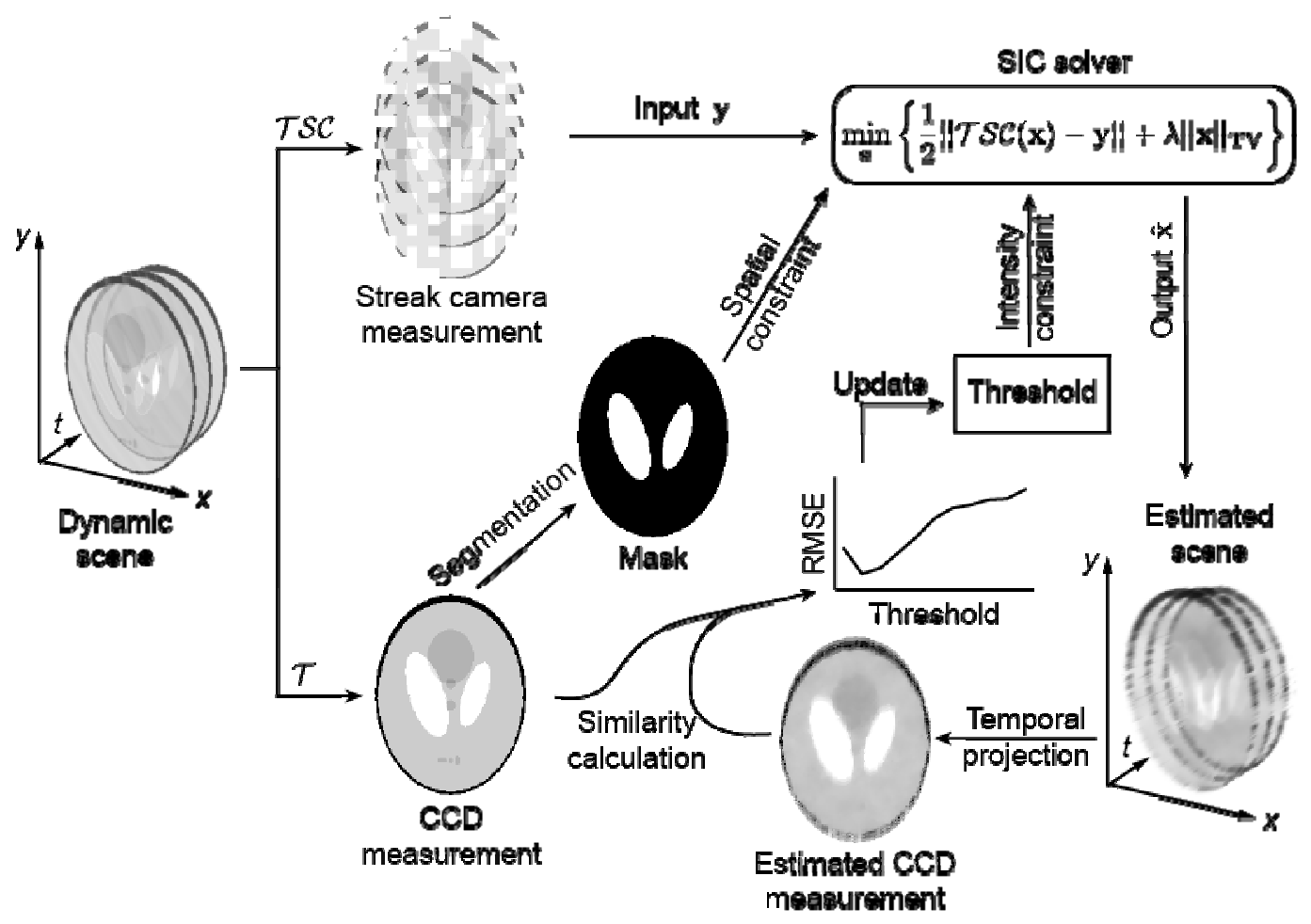

Figure 1. Operation principle of the spatially and intensity constrained (SIC) reconstruction. 
In our proposed method (outlined in Fig. 1), an external CCD camera records an unsheared spatiotemporally integrated image of the dynamic scene. Without the blurring caused by the temporal shearing in the streak tube, this additional perspective on the dynamic scene enjoys a better spatial resolution. To leverage this advantage, we first extract a spatial mask from the time-unsheared image, which is later used to define the zone of action in image reconstruction. A fairly standard grayscale segmentation approach is employed in this step: First, an adaptive local thresholding algorithm ${ }^{17}$ is applied to the image with a 15-pixel circular local window, to get an initial binary mask. Then, a 5-pixel median filter is applied to remove salt-and-pepper noise on the binary image. The resultant 2D binary mask is then used as a spatial constraint in the optimization framework, so that pixels outside the mask are not updated during optimization and remain zero. Such a spatial constraint improves the spatial resolution of the reconstructed datacube and accelerates the reconstruction procedure by reducing the degrees-of-freedom of the underlying object function.

Second, to reduce the low-intensity artifacts in the reconstructed datacube, we introduce an intensity threshold constraint, based on the time-unsheared image, in the optimization algorithm. Taking advantage of the fact that an iterative shrinkage/thresholding (IST) optimizer can impose convex set constraints at each iteration without losing its convergence properties ${ }^{18}$, we apply an intensity threshold to the optimization problem in Eq. 1 . The optimal threshold is chosen based on the similarity between the external CCD camera image and the temporally integrated image of the reconstructed datacube. We use the root-mean-square error (RMSE) as the similarity criterion. Mathematically, if we denote $\mathbf{y}_{0}$ as the measured external CCD camera image and $\mathbf{x}_{s}$ as the optimized solution with a threshold $s$, the optimal threshold is chosen by the following formula:

$$
\hat{s}=\arg \min _{s} \sqrt{\frac{1}{N}\left\|\mathcal{T}\left(\mathbf{x}_{s}\right)-\mathbf{y}_{0}\right\|_{2}^{2}},
$$

where the objective function is the RMSE, and $N$ is the total number of pixels in $\mathbf{y}_{0}$. Since this optimization problem is based on a separate parameterized optimization problem (i.e. solving for $\mathbf{x}_{s}$ with a given $s$ ), common gradient-based minimization methods become ineffective. We therefore employ a simple grid search method to find an approximation. More specifically, we first solve Eq. 1 with $s=0$, and then find the largest pixel value in the solution. Values between 0 and 0.01 of this maximum pixel value are considered candidates for the optimal threshold. Eleven evenly distributed threshold values are then tried, and the RMSE criteria are calculated for each threshold. The result for the minimal RMSE is then chosen as the final reconstruction result.

Since multiple intermediate solutions of Eq. 1 are needed in our method, a fast algorithm for acquiring one solution to Eq. 1 becomes more desirable for SIC reconstruction. The fast iterative shrinkage/thresholding algorithm (FISTA) ${ }^{18}$ has a reportedly faster convergence rate than the previously employed TwIST algorithm in solving general TV-regularized $\ell^{2}$-norm minimization problems, such as the one stated in Eq. 1, and hence is used in SIC reconstruction. To further accelerate our reconstruction method, we also implement the algorithm with the CUDA parallel programming framework on a single Tesla K40c graphic processing unit (GPU), reducing typical reconstruction time from tens of minutes to seconds.

\section{NUMERICAL SIMULATION}

We first validated our reconstruction method on numerically simulated data. A 200-by-200 Shepp-Logan (S-L) phantom was used as the base image. The simulated dynamic scene contained 10 frames, with the $2^{\text {nd }}, 5^{\text {th }}$, and $8^{\text {th }}$ frames set as the S-L phantom and the other frames left black (set to zeroes). The streak camera measurement was generated according to the forward model, and 1\% Gaussian white noise was added. Similarly, the CCD measurement was generated by integrating the original dynamic scene along the time axis. To demonstrate the advantages of our method, the dynamic scene was reconstructed using both the conventional TwIST-based unconstrained reconstruction method and our proposed SIC reconstruction method.

Figure 2 shows the simulation results. The similarity metric varies when we tune the intensity threshold value, as shown in Fig. 2 (a). In this particular case, a threshold of 0.006 gives us the best similarity, and the datacube generated by this threshold is chosen to be the final reconstruction result. Figure 2 (b), (c), and (d) show the same frame (the $2^{\text {nd }}$ frame) of the ground truth (the base image), the TwIST reconstructed result, and the SIC reconstructed result. Focusing on region 1, the large bright patch demonstrates better contrast in the image produced by the SIC reconstruction than that by the conventional TwIST reconstruction. In region 2, the SIC method successfully recovers the small bright spot present in 
the ground truth, while the conventional TwIST method fails to reconstruct this feature. The boundary between the dark and bright patches in region 3 is also more prominent in our results than in the TwIST result.
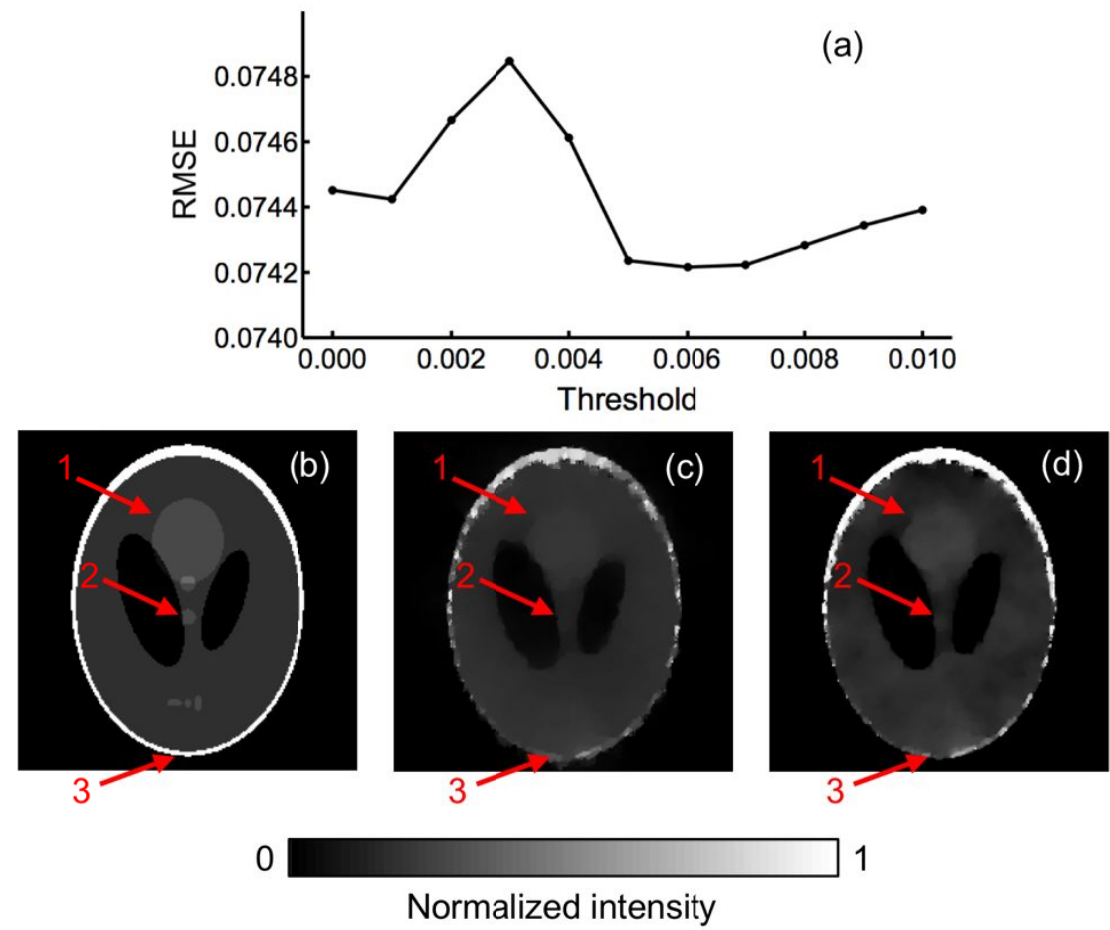

Figure 2. Results of the numerical simulation. (a) Root-mean-square error (RMSE) as the similarity metric versus tested threshold values. (b) The $2^{\text {nd }}$ frame of the ground truth dynamic scene. (c) as (b), but shows the TwIST reconstructed result. (d), as (b) and (c), but shows the SIC reconstructed result.

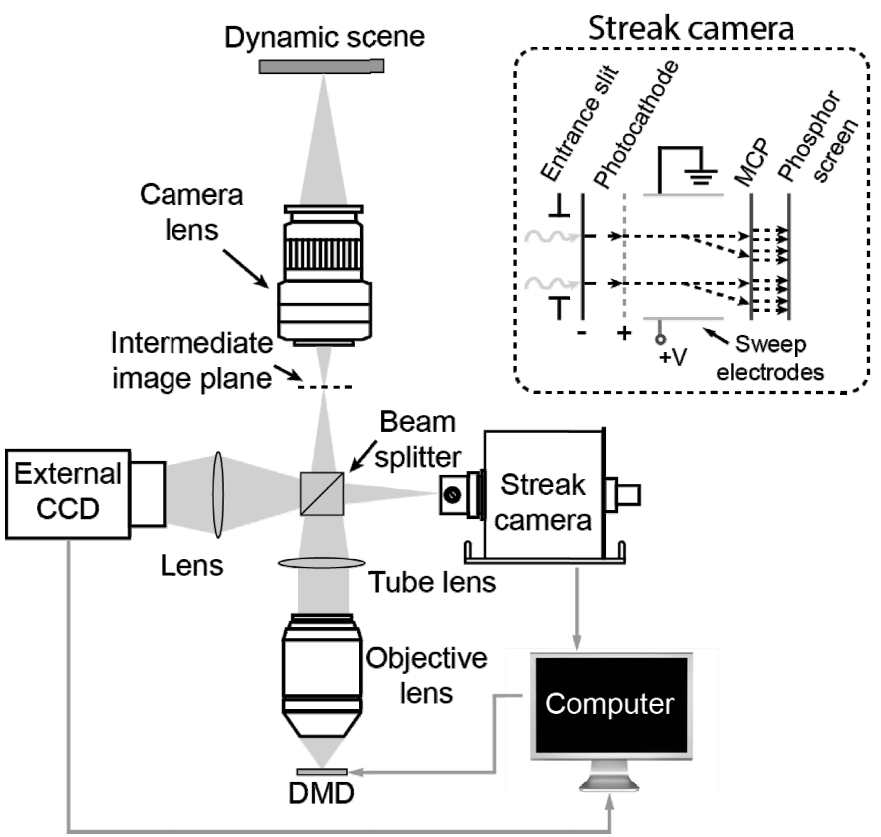

Figure 3. Schematic of the CUP system. Inset is a detailed illustration of the principles of the streak camera. DMD, digital micromirror device; MCP, microchannel plate; V, sweeping voltage. Equipment details: camera lens, Nikon, $\mathrm{f}=18-55 \mathrm{~mm}$; external CCD camera, Point Grey, GS3-U3-28S4M-C; internal CCD, Hamamatsu ORCA-R2; objective lens, Olympus, UPLSAPO 4×; streak camera, Hamamatsu, C7700; tube lens, Thorlabs, AC254-150-A. 


\section{EXPERIMENTAL RESULTS}

To experimentally validate our method, we upgraded the first-generation CUP system as shown in the system schematic, Fig. 3. The dynamic scene is first imaged by a camera zoom lens (focal length $18-55 \mathrm{~mm}$ ) to an intermediate image plane. Then a beam splitter divides the light into two directions. The reflected light is directly imaged by an external CCD camera. The transmitted light is passed to a DMD by a $4 f$ imaging system with a tube lens and an objective lens. A pseudorandom-binary pattern is programmed on the DMD to spatially encode the dynamic scene. Collected by the same objective lens, the encoded scene is further imaged to the entrance slit of a streak camera. Inside the streak camera, the incident light is first converted into photoelectrons by a photocathode. After initial acceleration, these photoelectrons are sheared by a sweeping voltage in the vertical axis, according to the time of flight (inset in Fig. 3). Then, the temporally sheared photoelectrons bombard a microchannel plate (MCP), where the current is amplified by generating secondary electrons. A phosphor screen converts the electrons back into light. An internal CCD camera then images the phosphor screen and compressively records the spatially encoded, temporally sheared dynamic scene in a single 2D image.

With this upgraded CUP system, we imaged a dynamic scene, where a laser pulse $(532 \mathrm{~nm}$ wavelength, 7 ps pulse duration) swept across a car-model target. The laser beam was first passed through an engineered diffuser and illuminated the target at an oblique angle of $\sim 30$ degrees with respect to the surface normal. The CUP system was placed perpendicular to the target's surface to collect the scattering photons. The system speed was 100 billion frames per second, achieved by setting the streak camera's shearing velocity to $1.32 \mathrm{~mm} / \mathrm{ns}$.

Similar to the simulation study, we reconstructed the dynamic scene using both the unconstrained TwIST method and the new SIC reconstruction method. Figure 4 (a) and (b) show temporally projected images of the reconstructed datacubes. The results of the proposed method illustrate sharper boundaries. Specifically, the line profiles plotted in Fig. 4 (c) show that the gaps between the wheels and the car body, while fuzzy in the TwIST result, become clear in the SIC result, and there are fewer artifacts within the object.
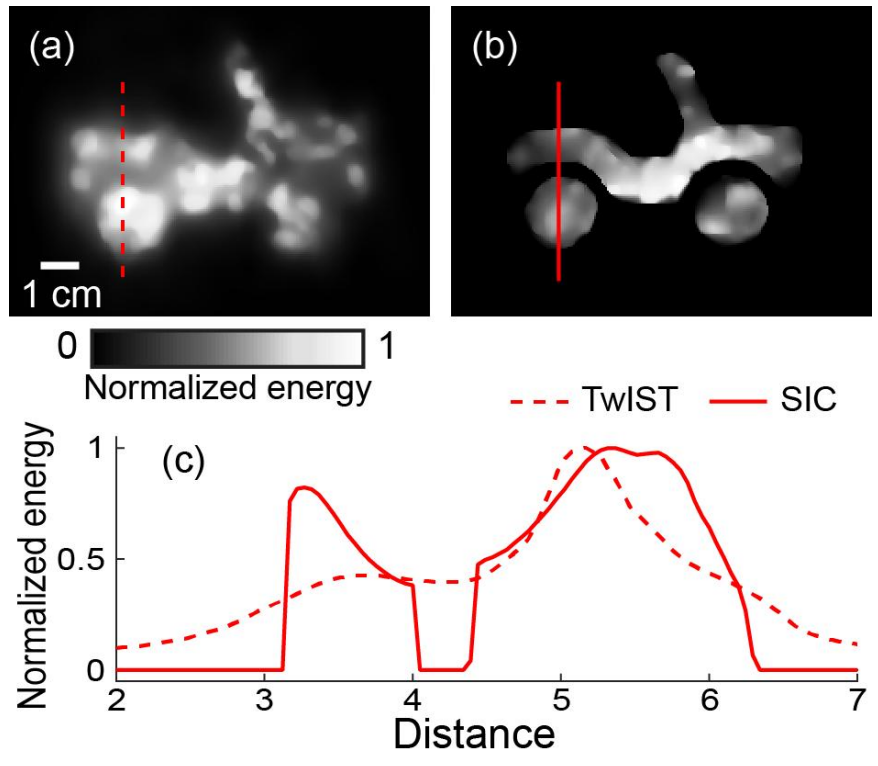

Figure 4. Experimental results. (a) Temporally projected image of the TwIST-reconstructed datacube. (b) The same projected image from the SIC-reconstructed datacube. (c) The profiles along lines indicated in (a) and (b).

\section{DISCUSSION AND CONCLUSIONS}

Our previous ToF-CUP technique ${ }^{15}$ uses a similar system setup, with one streak camera channel and one external CCD channel. However, ToF-CUP reconstruction only overlays the grayscale time-unsheared image on the TwISTreconstructed datacube as a post-processing method. The SIC method, on the contrary, extracts a 2D mask and 
incorporates it as a spatial constraint in the optimizer. Moreover, by adding and optimizing an intensity constraint, SIC finds an adaptive spatiotemporal mask based on the recovered datacube. The combined effect of spatial and intensity constraints not only eliminated artifacts in the expected black regions, but also improved image quality inside the zone of action, as demonstrated in both simulated and experimental results. Naturally, by applying the SIC reconstruction method to the ToF-CUP system, one can expect even more accurate ToF information across the object surface, with better spatial resolution.

In conclusion, our SIC reconstruction method incorporates an external CCD camera that captures an alternative perspective of the dynamic scene. Our new method exploits the resultant additional prior information by extracting spatial and intensity constraints from the external CCD image. As demonstrated with both numerical simulation and experimental data, the SIC reconstruction method recovers the dynamic scene with shaper boundaries, higher feature contrast, fewer low-intensity artifacts, and, in general, better image quality than the unconstrained TwIST reconstruction method in previous CUP technologies. Although it requires solving the original optimization problem multiple times, our SIC reconstruction leverages a faster reconstruction algorithm as well as current advances in computational hardware and GPU parallel computing technologies to reduce reconstruction time.

\section{ACKNOWLEDGEMENT}

The authors would like to thank Prof. James Ballard for reading the manuscript. This work was supported in part by National Institutes of Health grants DP1 EB016986 (NIH Director's Pioneer Award) and R01 CA186567 (NIH Director's Transformative Research Award).

\section{REFERENCES}

[1] Fuller, P.W.W., "An introduction to high speed photography and photonics,” The Imaging Science Journal 57(6), 293-302 (2009).

[2] Invisible Vision Ltd., "UBSi - True 1 billion fps ultra high speed framing camera," invisiblevision.com, $<$ http://www.invisiblevision.com/pdf/UBSi_(1Bn_fps_Camera).pdf $>$ (8 January 2016).

[3] Velten, A., Willwacher, T., Gupta, O., Veeraraghavan, A., Bawendi, M.G., and Raskar, R., "Recovering threedimensional shape around a corner using ultrafast time-of-flight imaging," Nature Communications 3, 745 (2012).

[4] Schaffer, C.B., Nishimura, N., Glezer, E.N., Kim, A.M.T., and Mazur, E., "Dynamics of femtosecond laserinduced breakdown in water from femtoseconds to microseconds," Optics Express 10(3), 196-203 (2002).

[5] Gariepy, G., Krstajić, N., Henderson, R., Li, C., Thomson, R.R., Buller, G.S., Heshmat, B., Raskar, R., Leach, J., et al., "Single-photon sensitive light-in-fight imaging," Nature Communications 6, 6021 (2015).

[6] Stolow, A., Bragg, A.E., and Neumark, D.M., "Femtosecond time-resolved photoelectron spectroscopy," Chemical Reviews 104(4), 1719-1758 (2004).

[7] Hockett, P., Bisgaard, C.Z., Clarkin, O.J., and Stolow, A., "Time-resolved imaging of purely valence-electron dynamics during a chemical reaction," Nature Physics 7(8), 612-615 (2011).

[8] Trebino, R., DeLong, K.W., Fittinghoff, D.N., Sweetser, J.N., Krumbügel, M.A., Richman, B.A., and Kane, D.J., "Measuring ultrashort laser pulses in the time-frequency domain using frequency-resolved optical gating," Review of Scientific Instruments 68(9), 3277-3295 (1997).

[9] Li, Z., Zgadzaj, R., Wang, X., Chang, Y.-Y., and Downer, M.C., "Single-shot tomographic movies of evolving light-velocity objects," Nature Communications 5, 3085 (2014).

[10] Nakagawa, K., Iwasaki, A., Oishi, Y., Horisaki, R., Tsukamoto, A., Nakamura, A., Hirosawa, K., Liao, H., Ushida, T., et al., "Sequentially timed all-optical mapping photography (STAMP)," Nature Photonics 8(9), 695-700 (2014).

[11] Goda, K., Tsia, K.K., and Jalali, B., "Serial time-encoded amplified imaging for real-time observation of fast dynamic phenomena," Nature 458(7242), 1145-1149 (2009).

[12] Heshmat, B., Satat, G., Barsi, C., and Raskar, R., "Single-shot ultrafast imaging using parallax-free alignment with a tilted lenslet array," presented at CLEO, 8 June 2014, San Jose, STu3E.7.

[13] Shiraga, H., Nakasuji, M., Heya, M., and Miyanaga, N., "Two-dimensional sampling-image x-ray streak camera for ultrafast imaging of inertial confinement fusion plasmas," Review of Scientific Instruments 70(1), 620-623 (1999).

[14] Gao, L., Liang, J., Li, C., and Wang, L.V., "Single-shot compressed ultrafast photography at one hundred billion frames per second," Nature 516(7529), 74-77 (2014). 
[15] Liang, J., Gao, L., Hai, P., Li, C., and Wang, L.V., "Encrypted Three-dimensional Dynamic Imaging using Snapshot Time-of-flight Compressed Ultrafast Photography," Scientific Reports 5, 15504 (2015).

[16] Bioucas-Dias, J.M., and Figueiredo, M.A.T., "A New TwIST: Two-Step Iterative Shrinkage/Thresholding Algorithms for Image Restoration,” IEEE Transactions on Image Processing 16(12), 2992-3004 (2007).

[17] Phansalkar, N., More, S., Sabale, A., and Joshi, M., "Adaptive local thresholding for detection of nuclei in diversity stained cytology images," presented at International Conference on Communications and Signal Processing, 2011, 218-220.

[18] Beck, A., and Teboulle, M., "Fast Gradient-Based Algorithms for Constrained Total Variation Image Denoising and Deblurring Problems," IEEE Transactions on Image Processing 18(11), 2419-2434 (2009). 